\title{
Otimismo Disposicional, Afetos e Personalidade em Pacientes com Doença Renal Crônica
}

\author{
Renata Cipriano de Oliveira ${ }^{1}$ \\ Joaquim Carlos Rossini ${ }^{1}$ \\ ${ }^{1}$ Universidade Federal de Uberlândia, MG, Brasil. \\ ${ }^{1}$ Universidade Federal de Uberlândia, MG, Brasil. \\ Renata Ferrarez Fernandes Lopes ${ }^{1}$ \\ ${ }^{1}$ Universidade Federal de Uberlândia, MG, Brasil.
}

Resumo: Este estudo investiga relações entre otimismo disposicional, afetos e traços de personalidade em pacientes com doença renal crônica que aguardam por um transplante renal (grupo pré-transplante) e em pacientes que já fizeram o procedimento (grupo pós-transplante). Teve como objetivos específicos: verificar se houve diferença nos escores desses três constructos nas duas amostras; se houve diferenças de desempenho no teste de otimismo e na escala de afetos em função do tempo de transplante; e se houve diferenças de desempenho no teste de otimismo e na escala de afetos em função do tempo de espera em lista. A pesquisa contou com quinze participantes em cada grupo sendo usados quatro instrumentos para coleta de dados: questionário sociodemográfico, Teste para avaliar o Otimismo (LOT-R), Inventário dos Cinco Grandes Fatores (NEO-FFI-R) e Escala de Afetos Positivos e Afetos Negativos (Panas). Os resultados indicaram maior otimismo nos participantes do grupo Pré-transplante ( $M d=29$; $I I Q=2)$ comparado com os do grupo Pós-transplante $(M d=26 ; I I Q=1)$. Conclui-se que no grupo pré-transplante, o otimismo disposicional não se associou de maneira significativa com nenhum fator de personalidade, o que pode indicar que o otimismo esteja voltado para um objetivo-alvo, mais do que para expectativas gerais de que coisas boas aconteçam e se evidencia como fator de proteção da personalidade, ou como mecanismo de ajustamento à condição crítica de hemodiálise. O tempo passado em lista de espera e de realização do transplante não tiveram associação significativa às demais variáveis.

Palavras-chave: Otimismo Disposicional, Afetos, Personalidade, Doença Renal Crônica.

\section{Dispositional Optimism, Affects and Personality in Patients with Chronic Renal Disease}

\begin{abstract}
This study investigated the relationship between dispositional optimism, affects and personality traits in patients with Chronic Renal Disease who are waiting for a renal transplant (Pre-Transplant Group) and in patients who have already undergone the procedure (PostTransplant Group). The specifics aims were to evaluate differences in construct scores between the groups, differences in performance on the optimism test and the affect scale associated with the waiting time in list, and differences in performance on the optimism test and the affect scale associated with the time of transplantation. Thirty people participated in the survey (fifteen in each group) and four instruments were used for data collection: sociodemographic questionnaire, Life Orientation Test (LOT-R), Five-Factor Inventory (NEO-FFI-R), and Positive and Negative Affect Schedule (PANAS). The results indicated greater optimism among the participants in the pre-transplant group $(M d=29, I Q R=2)$ compared to those in the post-
\end{abstract}


transplant group ( $M d=26, I Q R=1)$. In the pre-transplant group, dispositional optimism was not significantly associated with any personality factor, which may indicate that optimism in this group is focused on a target objective rather than to general expectations that good things happen. Optimism overlaps with the general characteristics of the subject and is evidenced as a personality protection factor, or as a mechanism of adjustment to the critical condition of hemodialysis. The waiting list time, as well as the time of transplantation, did not show a significant association with the other variables.

Keywords: Dispositional Optimism, Affects, Personality, Chronic Kidney Disease.

\title{
Optimismo Disposicional, Afectos y Personalidad en Pacientes con Enfermedad Renal Crónica
}

\begin{abstract}
Resumen: Este estudio objetivó investigar relaciones entre optimismo disposicional, afectos y rasgos de personalidad en pacientes con enfermedad renal crónica que esperan un trasplante renal (grupo pretrasplante) y en pacientes que ya fueron sometidos al procedimiento (grupo postrasplante). Los objetivos específicos fueron: evaluar si hay diferencia de puntuaciones de los constructos entre los grupos; si hay diferencias de desempeño en la prueba de optimismo y la escala de afectos en función del tiempo de trasplante; y si hay diferencias de desempeño en el test de optimismo y en la escala en función del tiempo de espera en la lista. La investigación tuvo quince participantes en cada grupo y se utilizaron cuatro instrumentos para recopilar los datos: cuestionario sociodemográfico, Test de Optimismo (LOT-R), Inventario de los Cinco Grandes Factores (NEO-FFI-R) y Escala de Afectos Positivos y Afectos Negativos (PANAS). Los resultados mostraron mayor optimismo en el grupo pretransplante $(M d=29, I I Q=2)$ en comparación con el grupo postransplante $(M d=26, I I Q=1)$. Se concluye que, en el grupo pretrasplante, el optimismo disposicional no se asoció de manera significativa con ningún factor de personalidad, lo que puede indicar que el optimismo esté más orientado hacia un objetivo que hacia expectativas generales de que cosas buenas pueden suceder y se evidencia como un factor de protección de la personalidad, o como un mecanismo de ajuste a la condición crítica de hemodiálisis. El tempo en la lista de espera y de realización de trasplante no mostraron asociación significativa con las demás variables.
\end{abstract}

Palabras clave: Optimismo Disposicional, Afectos, Personalidad, Enfermedad Renal Crónica.

\section{Introdução}

A Doença Renal Crônica (DRC) é considerada problema de saúde pública mundial, além disso, a incidência de problemas relacionados à função renal tem aumentado na população brasileira, assim como os custos para o seu tratamento (Bastos, Bregman, \& Kirsztajn, 2010). Por definição, esta doença consiste em "lesão renal e perda progressiva e irreversível da função dos rins (glomerular, tubular e endócrina)" (Romão, 2004, p. 1).

A condição de DRC se estabelece em estágios: fase de lesão com função renal normal, fase de insuficiência renal funcional ou leve, fase de insuficiência renal laboratorial ou moderada, fase de insuficiência renal clínica ou severa e fase terminal de insuficiência renal crônica. No último estágio de evolução da doença, Insuficiência Renal Crônica (IRC), as funções renais encontram-se bastante alteradas, colocando o portador da doença em risco de morte. Neste estágio, o paciente fica bastante sintomático, podendo apresentar anemia, desnutrição, problemas cardiorrespiratórios, entre outros (Bastos \& Kirsztajn, 2011; Romão, 2004).

$\mathrm{Na}$ fase de IRC, o paciente tem como opções de tratamento as Terapias Renais Substitutivas (TRS) que consistem nos métodos de depuração artificial do sangue (diálise peritoneal ou hemodiálise) ou no 
transplante renal. Dentre as opções de TRS, a hemodiálise é o tratamento dialítico mais utilizado atualmente no Brasil. Segundo Sesso, Lopes, Thomé, Lugon e Martins (2017), no país, 92\% dos pacientes com IRC estavam em hemodiálise, $8 \%$ em diálise peritoneal e $24 \%$ estavam em fila de espera para transplante. A hemodiálise é um processo que permite a filtragem e depuração do sangue de substâncias indesejáveis, como a creatinina e a ureia, que necessitam ser eliminadas da corrente sanguínea humana, já que este mecanismo é deficiente nos pacientes portadores de IRC (Nascimento \& Marques, 2005). Neste procedimento, a filtração extracorpórea do sangue é realizada por uma máquina, sendo necessário que o portador da IRC compareça aos centros de diálise em geral três vezes por semana para sessões que podem ter de duas a seis horas de duração (Kusumoto, Marques, Haas, \& Rodrigues, 2007).

Ainda que a hemodiálise aumente a sobrevida do paciente com insuficiência renal, uma vez que viabiliza o desempenho de uma função vital, impõe ao mesmo tempo limitações significativas. A vivência das doenças crônicas, em geral, pode ocasionar prejuízo no desempenho das atividades cotidianas associado a um declínio da vitalidade, bem como mudança significativa nos relacionamentos afetivos (Stanton, Revenson, \& Tennen, 2007). No caso específico da doença renal, ocorrem mudanças e perdas biopsicossociais, como perda ou dificuldade de manter atividades remuneradas, alterações na imagem corporal, restrições dietéticas e hídricas (Martins \& Cesarino, 2005).

Além disso, os pacientes que necessitam de transplante transitam entre a expectativa de ter a vida renovada e a possibilidade de morte iminente. A espera por um órgão, muitas vezes longa, quando não culmina em óbito do paciente, pode gerar distúrbios psicológicos (Machado, 2006). Esses pacientes estão, assim, suscetíveis a pior qualidade de vida, quando comparados à população geral, e maior prevalência de transtornos de humor (Barbosa, Andrade, \& Bastos, 2007; Stasiak, Bazan, Kuss, Schuinski, \& Baroni, 2014; Thomas \& Alchieri, 2005). A depressão é a complicação mais comum em pessoas em diálise, e pode significar uma resposta a alguma perda real ou imaginada (Georgianni \& Babatisikou, 2014). Os pacientes tendem a apresentar humor depressivo persistente, autoimagem prejudicada e sentimentos pessimistas (Higa, Kost, Soares, Morais, \& Polins, 2008). Diante disso, um tratamento que vise não somente suprir a deficiência renal, mas que aborde também aspectos psicossociais pode impactar positivamente na qualidade de vida do paciente renal crônico.

Uma alternativa aos métodos de depuração artificial do sangue é o transplante renal, através do qual o rim do paciente é substituído pelo rim de um doador, podendo ser este vivo ou falecido. $\mathrm{O}$ transplante é apontado como uma das modalidades de tratamento e reabilitação mais recomendadas, pois pode oferecer melhor qualidade de vida ao paciente, uma possível redução do risco de mortalidade e um custo econômico reduzido se comparado à diálise (Cunha et al., 2007). Sendo assim, em uma situação ideal, o transplante renal seria a melhor opção de TRS, já que poderia trazer menos impacto e/ou limitações para o funcionamento geral do indivíduo.

Rebollo et al. (2000) realizaram um estudo testando a Qualidade de Vida Relacionada à Saúde (QVRS) em pacientes em hemodiálise e em outros que já haviam sido transplantados. Em seus resultados, a qualidade de vida desses últimos apresentava-se melhor que no primeiro grupo e em similaridade com a população em geral. Pereira et al. (2003) e Bittencourt, Alves Filho, Mazzali e Santos (2004) também encontraram resultados semelhantes. Tais observações corroboram a expectativa generalizada de que o transplante seja um ganho para o indivíduo com doença renal.

Por outro lado, a realização do transplante pode não atender às expectativas almejadas. Pacientes transplantados podem ter vivenciado um longo tempo em lista de espera a ponto de suas condições físicas e emocionais terem sido muito deterioradas, ou mesmo passarem pelo processo de rejeição do enxerto transplantado (Machado, 2006). Ravagnani, Domingos e Miyazaki (2007) realizaram um estudo sobre qualidade de vida e enfrentamento realizado com a participação de 17 pacientes que responderam aos instrumentos da pesquisa (Inventário de Qualidade de Vida SF36 e o Inventário de Estratégias de Enfrentamento) em duas etapas: no período pré-transplante, quando em tratamento dialítico, e no pós-transplante, após um mínimo de três meses de enxerto funcionante. No que diz respeito à qualidade de vida, os resultados não mostraram melhoria estatisticamente significativa dos pacientes após o transplante renal.

Diante dessas observações é pertinente supor que outros fatores, que não apenas a realização do transplante, podem contribuir para uma efetiva melhora 
na qualidade de vida do paciente renal crônico, de modo que seria relevante que os pesquisadores em saúde se dedicassem a ampliar o conhecimento a respeito deles.

Conforme os estudos citados anteriormente, é notório o interesse por avaliar a presença e os efeitos de psicopatologias, como depressão e ansiedade, em pacientes renais crônicos. Embora tais pesquisas reconheçam a importância de conhecer o papel das síndromes sintomáticas sobre o paciente renal crônico, este estudo adotou uma perspectiva diferente, considerando a influência de aspectos positivos presentes no indivíduo e seu possível impacto no modo como ele se relaciona com as adversidades do tratamento. Para isso, os conceitos da Psicologia Positiva, em especial o construto do otimismo disposicional, foram fundamentais para investigar aspectos positivos da personalidade e suas relações com a condição da doença renal crônica.

$\mathrm{Na}$ visão da psicologia positiva, uma ciência mais ampla e uma prática mais completa em psicologia deveriam incluir a compreensão tanto do sofrimento quanto da felicidade, bem como a interação entre ambas. Nessa concepção são importantes as intervenções que venham a aliviar o sofrimento e, concomitantemente, aumentem a satisfação pessoal (Seligman, Steen, Park, \& Peterson, 2005). Um fenômeno psicológico importante abordado pela psicologia positiva é conhecido como "otimismo disposicional". O otimismo disposicional é definido como a tendência estável de um indivíduo em acreditar que coisas boas acontecerão, ao contrário de coisas ruins (Scheier \& Carver, 1985; Snyder \& Lopez, 2009). Deste ponto de vista, por acreditarem num futuro próspero, os otimistas tenderiam a empreender mais esforços para a transposição de obstáculos.

Scheier e Carver (1985) propuseram que o otimismo disposicional, sendo uma inclinação da personalidade que age nas bases da autorregulação do comportamento, levaria a várias consequências, incluindo algumas que seriam claramente relacionadas ao bem-estar físico e mental. Assim, para esses pesquisadores, o otimismo disposicional está associado com melhor saúde física e mental, menor preocupação com a severidade da doença, melhor habilidade funcional e maior bem-estar emocional (Scheier \& Carver, 1987). Carver e Scheier (2001) descreveram um modelo de autorregulação do comportamento, conhecido como expectancy-value model of motivation, segundo o qual o comportamento humano é organizado em função da busca de objetivos. A expectativa futura assume destaque nesta estrutura, pois está na base dos processos motivacionais que direcionam o indivíduo a atingir resultados ou evitar desfechos indesejáveis. De acordo com este modelo, as expectativas positivas sobre o futuro são importantes, possibilitando um senso de confiança de realização futura.

Bastianello e Hutz (2015) afirmam que ser otimista em tempos difíceis pode contribuir para redução no nível de ansiedade vivenciada pelo indivíduo, facilitando a manutenção de comportamentos positivos de adaptação. Vilhena et al. (2014), por sua vez, observam que os otimistas tendem a atitudes mais positivas e ao planejamento de ações que os levem a se recuperar de situações perturbadoras, a buscar mais informações e a ressignificar equilibradamente situações ruins. Além disso, é esperado que indivíduos otimistas apresentem predisposição a realizar ações confiantes e persistentes frente aos desafios da vida (Carver, Scheier, \& Segerstrom, 2010).

Alguns estudos têm sugerido que otimismo é um importante indicador de boa funcionalidade em pacientes que apresentam patologias crônicas, como artrite reumatoide (Brenner, Melamed, \& Panush, 1994), câncer de mama (Carver et al., 1993) e diabetes mellitus (Kavanagh, Gooley, \& Wilson, 1993). Contudo, não há ainda registros de estudos que associaram especificamente a DRC com o otimismo disposicional. Nesse sentido, e seguindo o que orientam Bastianello e Hutz (2015) quanto à importância de associar o otimismo a indicadores positivos do desenvolvimento, este estudo buscou investigar possíveis associações entre otimismo disposicional, afetos e traços de personalidade em uma amostra de pacientes renais crônicos.

Os afetos positivos e afetos negativos constituem a dimensão emocional do bem-estar subjetivo que, por sua vez, é a avaliação subjetiva que o indivíduo faz sobre sua situação no mundo. Representa uma avaliação pessoal sobre o quão feliz uma pessoa se sente de maneira independente ao contexto e outras variáveis que poderiam permitir uma avaliação objetiva de qualidade de vida (Nunes, Hutz, \& Giacomoni, 2009).

Sob o ponto de vista da psicologia positiva, os termos "afeto" e "emoção" (affect, emotion) e, algumas vezes, "humor" (mood) aparecem como sinônimos, não deixando a distinção conceitual tão clara (Lyubomirsky, King, \& Diener, 2005; Pressman \& 
Cohen, 2005). Para Fredrickson (2001), contudo, afeto é definido como um conceito generalizado que se refere a sentimentos conscientemente acessíveis, cujo efeito é mais duradouro, podendo ser flutuante e não emergir de um objeto determinado, sendo geralmente caracterizado por apenas duas dimensões emocionais, quais sejam a positiva e a negativa. As pessoas tendem, assim, a sentir afetos de maneira estável, o que não quer dizer que as emoções sejam imutáveis.

Os afetos podem ser caracterizados pela frequência e intensidade que os sujeitos estão propensos a sentir emoções positivas e negativas, como alegria, excitação, raiva e tristeza (Zanon, Bastianello, Pacico, \& Hutz, 2013b). Isso quer dizer que pessoas que experimentam frequentemente emoções positivas têm alto nível de afetos positivos. Por outro lado, as que sentem repetidamente emoções negativas apresentam níveis altos de afetos negativos. Ao se considerar o bem-estar, a frequência com que as pessoas vivenciam emoções positivas ou negativas é mais significativa do que a intensidade da experiência. Isso quer dizer que experimentar um número maior de emoções positivas, ainda que com menor intensidade, é mais relevante do que vivenciar uma emoção negativa de grande intensidade, como a perda de um ente querido. De maneira simples, aqueles que sentem mais emoções positivas, têm alta afetividade positiva e melhor bem-estar. De outro modo, pessoas que vivenciam emoções negativas com mais frequência, tendem à elevada afetividade negativa e redução na sensação bem-estar (Lyubomyrsky et al., 2005).

Os afetos positivos, bem como o bem-estar subjetivo, começaram a receber atenção nas pesquisas científicas com o avanço da psicologia positiva. Até então, os afetos positivos e negativos eram genericamente indiferenciados, sendo referenciados apenas como afetos ou emoções (Fredrickson, 1998, 2004). Também, emoções negativas recebiam mais destaque devido à sua função adaptativa ao longo da evolução da humanidade. As emoções negativas, como o medo, ocupavam o centro das discussões por desencadearem ações e comportamentos específicos de preservação (por exemplo, defesa ou fuga). As emoções positivas, por outro lado, eram pouco exploradas por serem associadas à falta de propósito ou inatividade (Fredrickson, 2004).

Dentro da nova perspectiva positiva, Lyubomirsky et al. (2005) pontuam que os afetos positivos relacionam-se a características como confiança, otimismo, autoeficácia, amabilidade, percepção positiva sobre os outros, sociabilidade, atividade, energia, comportamento pró-social e bem-estar físico, enfrentamento efetivo, originalidade e flexibilidade. Todos esses atributos têm em comum o fato de encorajar o indivíduo a desempenhar um envolvimento ativo em busca de objetivos, explorando o ambiente e vivenciando emoções positivas (Fredrickson, 2001). Assim, pessoas que experimentam os afetos positivos com mais frequência parecem conseguir ampliar seus recursos emocionais promovendo novas representações.

Nesse sentido, a personalidade é um dos mais importantes preditores de bem-estar subjetivo e é responsável pela característica de estabilidade que os afetos apresentam (Lucas \& Diener, 2008; Steel, Schmidt, \& Shultz, 2008). Lucas e Diener (2008) afirmam que os traços de personalidade do indivíduo são de grande importância no estudo dos afetos e do bem-estar subjetivo. São as características de personalidade de cada indivíduo que contribuem para que mantenha uma estabilidade afetiva ao longo da vida. Steel et al. (2008) defendem esta mesma ideia, de que a personalidade é um dos mais importantes preditores de bem-estar subjetivo. Para estes autores, a personalidade é responsável pela característica de estabilidade que os afetos apresentam. Assim, a frequência e intensidade com que os indivíduos sentem afetos positivos e negativos provavelmente dependem da forma como percebem e elaboram os eventos em suas vidas.

Um dos modelos mais utilizados no estudo dos aspectos da personalidade é o "modelo dos cinco grandes fatores de personalidade", conhecido como Big Five (McCrae \& Costa, 1997). Este é também o modelo de amplo consenso no campo de estudos da personalidade, graças aos mais de 50 anos de pesquisas sobre sua relevância e replicabilidade em diversas culturas (Hutz et al., 1998). As cinco dimensões da personalidade apresentadas neste modelo são: neuroticismo, extroversão, abertura à experiência, amabilidade e conscienciosidade. Cada uma delas é composta por facetas, que são características individuais mais específicas, como ansiedade, depressão, acolhimento, assertividade, confiança, altruísmo, competência e ponderação.

O estudo longitudinal de Costa e McCrae (1980) assumiu notoriedade no campo de investigação dos afetos, bem-estar subjetivo e personalidade. Essa investigação permite concluir que o fator extroversão, domínio que indica tendência à sociabilidade, é um 
bom indicador de afeto positivo, ao passo que o fator neuroticismo, domínio que avalia a suscetibilidade ao estresse, é indicador de afeto negativo. A partir daí, outros estudos foram realizados em âmbito internacional e chegaram a resultados similares. No Brasil, os trabalhos de Nunes et al. (2009) e Zanon, Bastianello, Pacico e Hutz (2013a) replicaram os mesmos achados.

Os fatores de personalidade também já apareceram associados ao otimismo disposicional em estudos como o de Williams (1992) e Boland e Cappeliez (1997) em que otimismo estava associado a baixos níveis de neuroticismo e altos níveis de extroversão. Marshall, Wortman, Kusulas, Hervig e Vickers (1992) realizaram também uma pesquisa em que avaliaram relações entre os cinco fatores de personalidade, otimismo e afetos. Os resultados revelaram que o pessimismo está associado de maneira positiva ao neuroticismo e afetos negativos e que o otimismo relaciona-se, também positivamente, com extroversão e afetos positivos.

O objetivo geral desta pesquisa foi analisar as relações entre otimismo disposicional, afetos e personalidade em duas amostras independentes, sendo uma de pacientes renais crônicos que aguardavam por um transplante renal e outra de pacientes que foram submetidos ao procedimento sem perda de enxerto. Os objetivos específicos foram: verificar se houve diferenças significativas nos escores de otimismo, afetos e traços de personalidade nas duas amostras; verificar se houve diferenças de desempenho no LOT-R e PANAS em função do tempo de transplante renal (no grupo de transplantados); verificar se houve diferenças de desempenho no LOT-R e PANAS em função do tempo de espera em lista (no grupo que aguarda por um transplante).

\section{Método}

\section{Participantes}

A amostra foi composta por conveniência e contou com a participação voluntária de 30 participantes que foram classificados em dois grupos independentes: grupo pré-transplante (15 participantes) e grupo pós-transplante (15 participantes). A Tabela 1 e a Tabela 2 mostram a caracterização da amostra de pacientes pré-transplante e pós-transplante conforme gênero, idade, escolaridade, religião, tempo de hemodiálise (grupo pré-transplante) e tempo de transplante (grupo pós-transplante).

\section{Tabela 1}

Caracterização geral do grupo pré-transplante.

\begin{tabular}{cccccc}
\hline Sujeito & Sexo & Idade & Escolaridade & Religião & Tempo de Hemodiálise (meses) \\
\hline 1 & M & 33 & Ensino Médio & Católica & 3 \\
2 & F & 23 & Ensino Superior Incompleto & Católica & 8 \\
3 & F & 56 & Ensino Fundamental & Evangélica & 72 \\
4 & M & 66 & Ensino Fundamental Incompleto & Espírita & 60 \\
5 & M & 55 & Ensino Superior & Católica & 60 \\
6 & M & 33 & Ensino Médio & Católica & 1 \\
7 & M & 56 & Ensino Superior & Nenhuma & 6 \\
8 & F & 26 & Ensino Superior Incompleto & Católica & 18 \\
9 & M & 42 & Ensino Médio & Católica & 204 \\
10 & F & 35 & Ensino Fundamental & Nenhuma & 132 \\
11 & F & 45 & Ensino Médio & Outra & 144 \\
12 & F & 47 & Ensino Fundamental & Católica & 18 \\
13 & F & 34 & Ensino Médio & Evangélica & 60 \\
14 & M & 62 & Ensino Fundamental & Católica & 144 \\
15 & M & 53 & Ensino Médio & Católica & 12 \\
\hline
\end{tabular}


Tabela 2

Caracterização geral do grupo pós-transplante.

\begin{tabular}{|c|c|c|c|c|c|}
\hline Sujeito & Sexo & Idade & Escolaridade & Religião & Tempo de Transplante (meses) \\
\hline 1 & $\mathrm{~F}$ & 23 & Ensino Médio & Evangélica & 16 \\
\hline 2 & M & 54 & Ensino Fundamental & Católica & 7 \\
\hline 3 & $\mathrm{~F}$ & 44 & $\begin{array}{l}\text { Ensino Fundamental } \\
\text { Incompleto }\end{array}$ & $\begin{array}{l}\text { Testemunha } \\
\text { de Jeová }\end{array}$ & 84 \\
\hline 4 & M & 46 & Ensino Fundamental & Evangélica & 2 \\
\hline 5 & M & 29 & Pós-Graduação & Espírita & 5 \\
\hline 6 & M & 55 & Ensino Médio & Espírita & 72 \\
\hline 7 & M & 47 & $\begin{array}{l}\text { Ensino Fundamental } \\
\text { Incompleto }\end{array}$ & Católica & 72 \\
\hline 8 & M & 65 & Ensino Fundamental & Nenhuma & 5 \\
\hline 9 & M & 51 & $\begin{array}{l}\text { Ensino Fundamental } \\
\text { Incompleto }\end{array}$ & Católica & 72 \\
\hline 10 & $\mathrm{~F}$ & 57 & Ensino Fundamental & Católica & 24 \\
\hline 11 & M & 54 & $\begin{array}{l}\text { Ensino Fundamental } \\
\text { Incompleto }\end{array}$ & Católica & 252 \\
\hline 12 & $\mathrm{~F}$ & 49 & Ensino Médio & Católica & 264 \\
\hline 13 & M & 31 & Ensino Médio & Católica & 3 \\
\hline 14 & M & 63 & Ensino Superior & Espírita & 36 \\
\hline 15 & $\mathrm{~F}$ & 41 & Ensino Médio & Espírita & 4 \\
\hline
\end{tabular}

Foram selecionados participantes com idade mínima de 18 anos e que não apresentavam comorbidades físicas e/ou psíquicas que pudesse inviabilizar a coleta de dados. Os componentes do grupo pré-transplante estavam em hemodiálise à espera de um doador de rim.

\section{Instrumentos}

Foram utilizados quatro instrumentos de avaliação, sendo um questionário sociodemográfico e três instrumentos psicológicos. O questionário sociodemográfico buscou identificar as características gerais da amostra: idade, sexo, grau de escolaridade, estado civil, tempo de transplante ou tempo de espera por transplante, religião e renda familiar.

Os três instrumentos psicológicos utilizados foram: a versão brasileira do Revised Life Orientation Test (LOT-R) composto por dez itens, sendo três sobre otimismo, três sobre pessimismo e quatro itens-filtro, cujos escores não são computados (Bastianello, Pacico, \& Hutz, 2014). O LOT-R apresenta boa consistência interna apresentando índices alfa de Cronbach variando de 0,70 a 0,80; 2); o Inventário dos Cinco Grandes Fatores (NEO-FFI-R) (FloresMendoza, 2007) composto por sessenta itens, divididos em cinco escalas (com 12 itens cada). A adaptação para o Brasil (Flores-Mendoza, 2007) apresenta boa consistência interna com um alfa de Cronbach igual a 0,81 para Neuroticismo; 0,78 para Extroversão; 0,74 para Abertura; 0,70 para Amabilidade e 0,83 para Conscienciosidade; 3) A Escala de Afetos Positivos e Afetos Negativos (PANAS) adaptada para o Brasil por Giacomoni e Hutz em 1997. Essa escala de autorrelato é composta por dez itens que avaliam afetos positivos e dez itens que avaliam afetos negativos avaliados por meio de uma escala do tipo Likert de cinco pontos.

\section{Procedimentos}

Todos os procedimentos éticos preconizados para esse tipo de investigação foram obedecidos e a pesquisa foi realizada após a aprovação no Comitê de Ética em Pesquisa (CEP), Protocolo: CAAE60779616.3.0000.5152. 
Os participantes do grupo pré-transplante foram convidados a participar da investigação quando se encontravam no setor de hemodiálise (no momento da diálise), enquanto os participantes do grupo pós-transplante foram localizados no ambulatório de transplante (na sala de espera para consulta) do Hospital de Clínicas da Universidade Federal de Uberlândia. Todos foram abordados e esclarecidos sobre os objetivos do estudo, ressaltando o caráter voluntário da participação. Os convidados que aceitaram participar receberam o Termo de Consentimento Livre e Esclarecido (TCLE) e, somente após o devido preenchimento e assinatura, responderam aos instrumentos específicos de pesquisa.

\section{Análise de dados}

Por se tratar de um estudo exploratório, os dados foram analisados por meio de estatística descritiva (média, desvio padrão, mediana e intervalo interquartil de amplitude), teste de probabilidade exata de Fisher e teste correlacional de Spearman. A comparação geral dos resultados obtidos nos dois grupos investigados foi realizada por meio do teste não paramétrico Mann-Whitney obtendo-se mediana e intervalo interquartil de amplitude de ambos os grupos. Utilizou-se o pacote estatístico SPSS, versão 20.0 com nível de significância $p \leq 0,05$.

\section{Resultados}

A idade média dos participantes do grupo pré-transplante foi de 44,4 anos $(D P=13,4)$. A idade média dos participantes do grupo pós-transplante foi igual a $47,3$ anos ( $\mathrm{DP}=12,1)$. O grupo pré-transplante foi composto por oito participantes do sexo masculino e sete participantes do sexo feminino. O grupo pós-transplante foi composto por dez participantes do sexo masculino e cinco participantes do sexo feminino. Uma análise por meio do teste de probabilidade exata de Fisher não evidenciou diferenças significativas quanto à frequência de participantes do sexo masculino e feminino que compuseram os dois grupos investigados, $p=0,71$. Quanto ao nível de escolaridade, $36,7 \%$ possuíam o ensino médio completo $(n=11), 23,3 \%$ tinham ensino fundamental completo $(n=7), 20 \%$ não concluíram o ensino fundamental $(n=6)$ e outros $20 \%$ ( $n=6)$ estavam entre nível superior e pós-graduação.

A Tabela 3 apresenta os resultados das correlações entre otimismo, Afetos Positivos (AP), Afetos Negativos (AN), os cinco fatores de personalidade (Neuroticismo - N, Extroversão - E, Abertura à Experiência - Ab, Amabilidade - Am e Conscienciosidade - C) e a idade dos participantes dos dois grupos, ou seja nos 30 indivíduos que responderam aos instrumentos da pesquisa.

\section{Tabela 3}

Correlação de Spearman entre Otimismo, Afetos Positivos, Afetos Negativos, Fatores de Personalidade e Idade de todos os participantes.

\begin{tabular}{cccccccccc}
\hline & Otimismo & AP & AN & N & E & Ab & Am & C & Idade \\
\hline Otimismo & 1,00 & & & & & & & & \\
AP & $0,37^{*}$ & 1,00 & & & & & & & \\
AN & $-0,31$ & 0,20 & 1,00 & & & & & & \\
N & $-0,41^{*}$ & $-0,07$ & $0,68^{*}$ & 1,00 & & & & & \\
E & $0,39^{*}$ & $0,45^{*}$ & 0,07 & $-0,25$ & 1,00 & & & & \\
Ab & 0,29 & $0,38^{*}$ & 0,02 & $-0,25$ & 0,22 & 1,00 & & & \\
Am & 0,24 & 0,15 & $-0,41^{*}$ & $-0,63^{*}$ & 0,10 & 0,10 & 1,00 & & \\
$\mathbf{C}$ & $0,36^{*}$ & $0,38^{*}$ & $-0,33$ & $-0,42^{*}$ & 0,24 & 0,08 & $0,38^{*}$ & 1,00 & \\
Idade & 0,21 & 0,21 & $-0,18$ & $-0,35$ & 0,06 & 0,08 & 010 & 0,34 & 1,00 \\
\hline${ }^{*} \mathrm{p}<0,05 ; \mathrm{N}=30$ & & & & & & & &
\end{tabular}

AP: Afetos Positivos; AN: Afetos Negativos; N: Neuroticismo; E: Extroversão; Ab: Abertura à Experiência; Am: Amabilidade; C: Conscienciosidade. 
Considerando a amostra total, o otimismo está correlacionado positivamente e de maneira significativa $(p<0,05)$ com os afetos positivos $(\rho=0,37)$, extroversão $(\rho=0,39)$ e conscienciosidade $(\rho=0,36)$ e de maneira negativa ao fator neuroticismo $(\rho=-0,41, p<0,05)$.

Afetos positivos apresentam relação significativa $(p<0,05)$ e positiva com extroversão $(\rho=0,45)$, abertura à experiência $(\rho=0,38)$ e conscienciosidade $(\rho=0,38)$. Já os afetos negativos no grupo total de pacientes renais crônicos estão relacionados de maneira positiva com o fator de personalidade neuroticismo $(\rho=0,68)$ e negativamente com amabilidade $(\rho=-0,41)$.
As associações entre as dimensões de personalidade evidenciam relações inversas (negativas) entre neuroticismo e amabilidade $(\rho=-0,64)$, neuroticismo e conscienciosidade $(\rho=-0,42)$, e relação positiva entre amabilidade e conscienciosidade $(\rho=0,38)$. Nesta primeira análise, a idade dos participantes não oferece nenhum indicativo relevante quando associada aos construtos investigados.

Buscando avaliar uma possível diferença no desempenho dos participantes do grupo pré e do grupo pós-transplante, os dados foram submetidos ao teste de Mann-Whitney. Os valores obtidos são observados na Tabela 4.

Tabela 4

Valores do teste de Mann-Whitney entre os grupos Pré e Pós-Transplantes.

\begin{tabular}{|c|c|c|c|c|c|}
\hline \multirow[b]{2}{*}{ Variáveis } & \multicolumn{2}{|l|}{ Mediana } & \multirow[b]{2}{*}{$\boldsymbol{U}$} & \multirow[b]{2}{*}{$Z$} & \multirow[b]{2}{*}{$\boldsymbol{P}$} \\
\hline & $\begin{array}{c}\text { Pré- } \\
\text { Transplante }\end{array}$ & $\begin{array}{c}\text { Pós- } \\
\text { Transplante }\end{array}$ & & & \\
\hline Otimismo & 29 & 26 & 63,5 & $-2,05$ & $0,04^{*}$ \\
\hline AP & 38 & 35 & 94 & $-0,77$ & 0,44 \\
\hline AN & 17 & 20 & 95 & $-0,73$ & 0,46 \\
\hline $\mathbf{N}$ & 41 & 42 & 99,5 & $-0,54$ & 0,59 \\
\hline $\mathbf{E}$ & 50 & 45 & 103,5 & $-0,37$ & 0,71 \\
\hline $\mathbf{A b}$ & 46 & 46 & 104 & $-0,35$ & 0,72 \\
\hline Am & 59 & 53 & 92 & $-0,85$ & 0,39 \\
\hline C & 59 & 56 & 89 & $-0,98$ & 0,32 \\
\hline
\end{tabular}

${ }^{*} \mathrm{p}<0,05 ; \mathrm{N}=15$

AP: Afetos Positivos; AN: Afetos Negativos; N: Neuroticismo; E: Extroversão; Ab: Abertura à Experiência; Am: Amabilidade; C: Conscienciosidade.

Os escores observados nos dois grupos confirmaram diferença significativa na escala LOT-R. Os indivíduos do grupo pré-transplante apresentaram valores significativamente maiores neste instrumento $(M d=29 ; I I Q=2)$ em comparação aos participantes do grupo pós-transplante $(M d=26 ; I I Q=1), U=63,5$ $(z=-2,05), p=0,04$.

Os escores das variáveis também foram analisados dentro de cada grupo especificamente. A Tabela 5 evidencia os resultados através do teste correlacional de Spearman no Grupo Pré-Transplante.

Neste grupo foram encontradas quatro relações significativas $(p<0,05)$. O fator de personalidade neuroticismo está relacionado de maneira positiva à AN $(\rho=0,75)$ mantendo assim o padrão encontrado na população geral, assim como no grupo total dos 30 participantes com doença renal crônica. De forma inversa, neuroticismo associa-se à amabilidade $(\rho=-0,53)$ e à idade dos participantes $(\rho=-0,56)$. É importante mencionar que o tempo em lista de espera não tem associação com os escores de otimismo e afetos. Por outro lado, o tempo apresentou relação positiva com o fator de personalidade amabilidade $(\rho=0,53)$. Otimismo disposicional não se relaciona de maneira relevante com nenhuma outra variável. Um número maior de relações significativas foi observado no grupo pós-transplante. Esses resultados são sumariados na Tabela 6 . 
Tabela 5

Correlação de Spearman entre otimismo, afetos positivos, afetos negativos, fatores de personalidade, tempo de hemodiálise (HD) e idade no grupo pré-transplante.

\begin{tabular}{|c|c|c|c|c|c|c|c|c|c|c|}
\hline & Otimismo & $\mathbf{A P}$ & AN & $\mathbf{N}$ & $\mathbf{E}$ & $\mathbf{A b}$ & Am & C & $\begin{array}{c}\text { Tempo } \\
\text { HD }\end{array}$ & Idade \\
\hline Otimismo & 1,00 & & & & & & & & & \\
\hline AP & 0,00 & 1,00 & & & & & & & & \\
\hline AN & $-0,10$ & 0,47 & 1,00 & & & & & & & \\
\hline $\mathbf{N}$ & $-0,13$ & 0,36 & $0,75^{*}$ & 1,00 & & & & & & \\
\hline $\mathbf{E}$ & 0,17 & 0,35 & 0,16 & 0,13 & 1,00 & & & & & \\
\hline $\mathbf{A b}$ & 0,13 & $-0,38$ & 0,06 & 0,06 & $-0,15$ & 1,00 & & & & \\
\hline Am & $-0,27$ & $-0,00$ & $-0,33$ & $-0,53^{*}$ & $-0,24$ & $-0,12$ & 1,00 & & & \\
\hline C & 0,11 & 0,41 & $-0,05$ & $-0,25$ & 0,25 & $-0,06$ & 0,41 & 1,00 & & \\
\hline Tempo HD & 0,03 & $-0,02$ & $-0,23$ & $-0,40$ & $-0,39$ & 0,15 & $0,53^{*}$ & $-0,03$ & 1,00 & \\
\hline Idade & 0,12 & $-0,08$ & $-0,39$ & $-0,56^{*}$ & $-0,10$ & $-0,19$ & 0,45 & 0,40 & - & 1,00 \\
\hline
\end{tabular}

AP: Afetos Positivos; AN: Afetos Negativos; N: Neuroticismo; E: Extroversão; Ab: Abertura à Experiência; Am: Amabilidade; C: Conscienciosidade.

Tabela 6

Correlação de Spearman entre otimismo, afetos positivos, afetos negativos, fatores de personalidade, tempo de transplante e idade no grupo pós-transplante.

\begin{tabular}{ccccccccccc}
\hline & Otimismo & AP & NA & N & E & Ab & Am & C & Tempo & Idade \\
\hline Otimismo & 1,00 & & & & & & & & & \\
AP & $0,72^{*}$ & 1,00 & & & & & & & & \\
AN & $-0,49$ & $-0,01$ & 1,00 & & & & & & & \\
$\mathbf{N}$ & $-0,67^{*}$ & $-0,37$ & 0,50 & 1,00 & & & & & & \\
E & 0,45 & $0,50^{*}$ & 0,04 & $-0,59^{*}$ & 1,00 & & & & & \\
Ab & 0,47 & $0,70^{*}$ & $-0,02$ & $-0,40$ & 0,36 & 1,00 & & & & \\
Am & $0,67^{*}$ & 019 & $-0,46$ & $-0,77^{*}$ & 0,41 & 0,25 & 1,00 & & & \\
$\mathbf{C}$ & $0,62^{*}$ & 0,30 & $-0,60^{*}$ & $-0,62^{*}$ & 0,22 & 0,18 & 0,46 & 1,00 & & \\
Tempo & 0,20 & 0,04 & $-0,10$ & $-0,04$ & 0,17 & 0,14 & 0,19 & 0,25 & 1,00 & \\
Idade & 0,29 & 0,49 & 0,06 & $-0,16$ & 0,03 & 0,29 & $-0,13$ & 0,38 & - & 1,00 \\
\hline${ }^{*} \mathrm{p}<0,05 ; \mathrm{N}=15$ & & & & & & & & &
\end{tabular}

AP: Afetos Positivos; AN: Afetos Negativos; N: Neuroticismo; E: Extroversão; Ab: Abertura à Experiência; Am: Amabilidade; $\mathrm{C}$ : Conscienciosidade.

O Otimismo está positivamente relacionado a AP $(\rho=0,72)$, amabilidade $(\rho=0,67)$ e conscienciosidade ( $\rho=0,62)$, e negativamente relacionado a dimensão neuroticismo $(\rho=-0,67)$. Ainda neste grupo, os AP estão associados de maneira positiva a extroversão $(\rho=0,50)$ e abertura à experiência $(\rho=0,70)$, enquanto os AN estão negativamente associados à conscienciosidade $(\rho=-0,60)$. $O$ fator neuroticismo apresentou 
relações negativas com as dimensões extroversão $(\rho=-0,59)$, amabilidade $(\rho=-0,77)$ e conscienciosidade $(\rho=-0,62)$. O otimismo vem associado de maneira negativa ao neuroticismo e apresenta relação positiva aos fatores amabilidade e conscienciosidade.

\section{Discussão}

Os resultados obtidos com a análise dos 30 participantes estão, de modo geral, em acordo com os achados de pesquisas anteriores (Marshall et al. 1992; Sharpe, Martin, \& Roth, 2011; Williams, 1992). O trabalho de Marshall et al. (1992) evidenciou associação positiva entre pessimismo, neuroticismo e afetos negativos e também associação positiva entre otimismo disposicional, extroversão e afetos positivos. Entre os 30 pacientes com doença renal crônica também apareceram relações semelhantes. Nesta amostra há uma tendência geral de que indivíduos com alto escore em neuroticismo apresentem níveis elevados de afetos negativos, assim como pacientes com altos níveis de extroversão tendem a apresentar escores elevados de otimismo, e também a experimentar mais afetos positivos.

Os resultados desta pesquisa também corroboram os obtidos por Sharpe et al. (2011). Neste estudo, os autores relacionaram otimismo e os cinco grandes fatores de personalidade obtendo evidências que sugerem que neuroticismo e extroversão são bons preditores do otimismo disposicional. A mesma investigação também sugere que os fatores amabilidade e conscienciosidade são características preditoras de otimismo. Isso significa dizer que as associações encontradas na amostra de portadores de DRC aproximam-se das encontradas na população em geral, e que os instrumentos usados atenderam à expectativa de medida proposta. As demais relações não encontram ainda suporte na literatura atual, merecendo melhor investigação.

A análise da diferença entre os grupos pré e pós-transplante por meio do teste de Mann-Whitney confirmou que os pacientes que aguardam em hemodiálise por um transplante renal tendem a ser mais otimistas, ou seja, eles têm maiores expectativas de que coisas boas acontecerão. No caso dos pacientes em fila de espera, é possível inferir que o otimismo esteja voltado para um objetivo-alvo, ou seja, a realização do transplante renal. Isto poderia explicar o fato de que sujeitos que já fizeram o transplante, tendo alcançado o objetivo-alvo, apresentem escores menores de otimismo disposicional.

Das correlações encontradas no grupo pré-transplante, vale a pena mencionar que o tempo de espera em hemodiálise não está relacionado a nenhuma das variáveis investigadas. Por outro lado, o tempo apresentou relação positiva com o fator de personalidade amabilidade $(\rho=0,53)$. Isto revela que, nesta amostra, quanto mais tempo o paciente aguarda por um transplante, mais características como empatia e cordialidade, se destacam. É possível supor que, com o passar do tempo, o agravamento da doença e mesmo os efeitos colaterais da terapia renal substitutiva levem à necessidade de que mais características de orientação interpessoal (facetas da amabilidade), como a receptividade, modéstia e a sensibilidade, surjam de forma mais evidente. A dependência de familiares, ou outras pessoas próximas, para o desempenho das atividades diárias pode ter ligação com essa particularidade.

Merece destaque nesta discussão o dado estatístico no qual o otimismo disposicional não se correlaciona de maneira significativa a nenhuma outra variável entre os pacientes que aguardam pelo transplante. Este resultado diverge dos resultados de literatura anteriores, segundo os quais o otimismo apresenta associação com os fatores de personalidade e com aspectos positivos da personalidade (Marshall et al., 1992; Sharpe et al., 2011; Vilhena et al., 2014). Nesse sentido, Vilhena et al. (2014) investigaram o papel do otimismo na qualidade de vida de pessoas portadoras de doenças crônicas e apontaram o otimismo enquanto mecanismo de ajustamento do indivíduo em condição de doença. Em seus resultados, o otimismo disposicional está correlacionado aos cinco fatores de personalidade e à melhor percepção do sujeito sobre sua saúde física e mental (aspectos da avaliação de qualidade de vida). Porém, neste estudo, entre os pacientes que aguardam em diálise pela possibilidade de realizar o transplante renal a variável otimismo não aparece associada a nenhuma outra. As características de personalidade dos sujeitos parecem não ser indicativas de maior ou menor otimismo.

O que leva a inferir que, para aqueles que estejam em hemodiálise, aguardando para realizar um transplante renal, o otimismo pode se sobrepor às características de personalidade como fator de proteção psicológica. Wrosch e Scheier (2003) destacam o papel que o otimismo disposicional desempenha como mecanismo adaptativo de autorregulação do 
comportamento, principalmente quando relacionado a circunstâncias críticas da vida. É razoável pensar que, em detrimento das peculiaridades da personalidade de cada indivíduo do grupo pré-transplante há uma necessidade maior do que no grupo pós-transplante de lançar mão do otimismo como importante no ajustamento à condição de lista de espera.

No grupo de pacientes transplantados, o otimismo disposicional tem a associação esperada entre alguns fatores da personalidade (neuroticismo, amabilidade e conscienciosidade) e afetos positivos. Para aqueles que alcançaram o objetivo de realizar o transplante, o otimismo mostra relações semelhantes às encontradas na população geral, em que os fatores de personalidade são variáveis indicativas de otimismo.

Assim, é possível supor que, para esses indivíduos, a expectativa (específica) de que algo bom aconteça perde força e os sujeitos precisem dispor de outros recursos para se adaptarem à nova condição (de transplantados). Ou ainda, é plausível pensar que esses indivíduos podem agora se ocupar de realizações que antes eram inviáveis pela necessidade de hemodiálise. As relações entre o tempo de realização de transplante e as demais variáveis também não se revelaram significativas.

\section{Considerações finais}

Os resultados obtidos a partir desta pesquisa contribuem com o campo da psicologia positiva e sua relação com a psicologia da saúde. Tendo em vista que pesquisas anteriores apontam para associações entre otimismo disposicional e saúde física e mental, foi oportuno aprofundar em uma condição crônica de saúde mais específica, que é a doença renal, a fim de se verificar como o otimismo e os afetos aparecem relacionados neste tipo de população.

Vale considerar que a pesquisa atendeu aos objetivos iniciais propostos e inicia uma agenda de pesquisa voltada à investigação qualitativa e quantitativa do otimismo disposicional e pacientes renais crôni$\cos$. Tais pesquisas podem esclarecer melhor a função que o otimismo assume para os pacientes que aguardam em lista de espera para transplante.

Por se tratar de uma amostra clínica, outros estudos poderiam avaliar o papel dos estilos de enfrentamento e suas relações com as variáveis estudadas nesta pesquisa relacionadas à DRC nos dois grupos. Esta sugestão parte das considerações de Scheier et al. (1989), segundo as quais os otimistas podem ter estratégias de enfrentamento para lidar com situações estressoras que promovam maior estado de bem-estar do que aquelas que são usadas por pessoas consideradas pessimistas. Outros estudos já buscaram associações entre otimismo e estilos de enfrentamento, como Scheier, Weintraub e Carver (1986), Scheier et al. (1989); Curbow, Somerfield, Baker, Wingard e Legro (1993); Botelho e Pereira (2015), o que pode ser útil no desenvolvimento de técnicas e estratégias de intervenção junto aos pacientes com DRC.

Por se tratar de um estudo exploratório, este trabalho conta com algumas limitações. Há a necessidade de ampliação da amostra investigada visando maior robustez na inferência populacional. Uma sugestão para futuros estudos é a adoção de um delineamento de pesquisa que avalie o mesmo paciente antes e depois do procedimento de transplante renal, o que poderá contribuir com informações mais amplas e precisas sobre o efeito das variáveis investigadas.

Outro fator a ser considerado é o uso do inventário de personalidade em sua versão breve, o NEO-FFI-R. O desempenho dos participantes neste instrumento não apontou para diferenças significativas entre os dois grupos no que se refere aos cinco grandes fatores de personalidade. Uma avaliação que considere aplicar a versão longa do inventário, o NEO-PI-R, permitiria que as facetas que compõem cada dimensão da personalidade, como ansiedade, depressão, acolhimento, assertividade, confiança, altruísmo e esforço por realizações, fossem melhor analisadas e relacionadas às variáveis centrais da pesquisa.

Para além das limitações apontadas acima, os resultados reforçam a necessidade de se considerar os aspectos psicológicos que envolvem o paciente crônico a fim de que intervenções que considere múltiplos fatores sejam feitas em favor dos pacientes. É possível hipotetizar que as abordagens em saúde multiprofissionais que envolvam não só o tratamento de reabilitação e recuperação física do paciente, mas que também considere o incremento das potencialidades e capacidades dos pacientes sejam mais eficazes. Para isso a psicoeducação da equipe sobre otimismo disposicional pode ser um primeiro passo para uma visão mais positiva do paciente em hemodiálise ou transplantado. Tal visão pode gerar práticas integrativas que estimulem o otimismo disposicional a partir de diferentes áreas de intervenção, inclusive da prática psicológica, que muitas vezes enfatiza a psicopatologia e a vulnerabilidade, em detrimento das capacidades e potencialidades do paciente. 
Neste caminho, os resultados desta pesquisa reforçam os preceitos da psicologia positiva ao evidenciar que, mesmo imerso em uma situação de vida dolorosa e limitante como a hemodiálise, o indivíduo é capaz de lançar mão de recursos positivos da personalidade, neste caso o otimismo disposicional, como amortecedor do risco psicológico, fator de proteção e resistência.

\section{Referências}

Barbosa, L. M. M., Andrade Junior, M. P., \& Bastos, K. A. (2007). Preditores de qualidade de vida em pacientes com doença renal crônica em hemodiálise. Jornal Brasileiro de Nefrologia, 29(4), 222-229.

Bastianello, M. R., \& Hutz, C. S. (2015). Do otimismo explicativo ao disposicional: A perspectiva da psicologia positiva. Psico-USF, 20(2), 237-247. http://doi:10.1590/1413-82712015200205

Bastianello, M. R., Pacico, J. C., \& Hutz, C. S. (2014). Optimism, self-esteem and personality: Adaptation and validation of the Brazilian version of The Revised Life Orientation Test (LOT-R). Psico-USF, 19(3), 523-531. http://doi:10.1590/1413-827120140190030

Bastos, M. G., Bregman, R., \& Kirsztjan, G. M. (2010). Doença renal crônica:Frequente egrave, mas também prevenível e tratável. Revista da Associação Médica Brasileira, 56(2), 248-253. http://doi:10.1590/S0104-42302010000200028

Bastos, M. G., \& Kirsztjan, G. M. (2011). Doença renal crônica: Importância do diagnóstico precoce, encaminhamento imediato e abordagem interdisciplinar estruturada para melhora do desfecho em pacientes ainda não submetidos à diálise. Jornal Brasileiro de Nefrologia, 33(1), 93-108. http://doi:10.1590/S0101-28002011000100013

Bittencourt, Z. Z. L. C., Alves Filho, G., Mazzali, M., \& Santos, N. R.(2004). Qualidade de vida em transplantados renais: Importância do enxerto funcionante. Revista de Saúde Pública, 38(5), 732-734. http://doi:10.1590/ S0034-89102004000500018

Boland, A., \& Cappeliez, P. (1997).Optimism and neuroticism as predictors of coping and adaptation in older women. Personality and Individual Differences, 22, 909-919. http://doi:10.1016/S0191-8869(96)00251-6

Botelho, A. S. F., \& Pereira, M. G. (2015). Qualidade de vida, otimismo, enfrentamento, morbidade psicológica e estresse familiar em pacientes com câncer colorrectal em quimioterapia. Estudos de Psicologia, 20(1), 50-60. http://doi:10.5935/1678-4669.20150007

Brenner, G. F; Melamed, B. G., \& Panush, R. S. (1994). Optimism and coping as determinants of psychosocial adjustment to rheumatoid arthritis. Journal of Clinical Psychology in Medical Settings, 1(2), 115-134. http://doi:10.1007/BF01999741

Carver, C. S., Pozo, C., Harris, S. D., Noriega, V., Scheier, M. F., Robinson, D. S., Ketcham, A. S., Moffat, F. L., Jr., \& Clark, K. C. (1993). How coping mediates the effect of optimism on distress: A study of women with early stage breast cancer. Journal of Personality and Social Psychology, 65, 375-390. http://doi:10.1037/0022-3514.65.2.375

Carver, C. S., \& Scheier, M. F. (2001). Optimism, pessimism and self-regulation. In C. Chang (Ed), Optimism and pessimism: Implications for theory, research and practice (pp. 31-51). Washington, DC: American Psychological Association. http://doi:10.1037/10385-002

Carver, C. S., Scheier, M. F., \& Segerstrom, S. S. (2010). Optimism. Clinical Psychology Review, 30, 879-889. http://doi:10.1016/j.cpr.2010.01.006

Costa, P. T., Jr., \& McCrae, R. R. (1980). Influence of extraversion and neuroticism on subjective well-being: Happy and unhappy people. Journal of Personality and Social Psychology, 38(4), 668-678. http://doi:10.1037/0022-3514.38.4.668

Cunha, C. B., León, A. C. P., Schramm, J. M. A., Carvalho, M. S., Souza, P. R. B., Jr., \& Chain, R. (2007). Tempo até o transplante e sobrevida em pacientes com insuficiência renal crônica no estado do Rio de Janeiro, Brasil 1998-2002. Cadernos de Saúde Pública, 23(4), 805-813. http://doi:10.1590/S0102-311X2007000400008

Curbow, B., Somerfield, M. R., Baker, F, Wingard, J. R., \& Legro, M. W. (1993). Personal changes, dispositional optimism, and psychological adjustment to bone narrow transplantation. Journal of Behavioral Medicine, 16(5), 423-441. http://doi:10.1007/BF00844815

Flores-Mendoza, C. E. (2007). Estudo brasileiro do NEO-FFI-R (versão curta). In P. T. Costa Junior \& R. R. McCrae, Avaliação em psicologia positiva NEO PI-R: Inventário de personalidade NEO revisado; e inventário de cinco fatores neo revisado: NEO-FFI-R (versão curta) (pp. 93-98). São Paulo, SP: Vetor.

Fredrickson, B. L. (1998). What good are positive emotions? Review of General Psychology, 2(3), 300-319. http://doi:10.1037/1089-2680.2.3.300 
Fredrickson, B. L. (2001). The role of positive emotions in positive psychology: The broaden-and-build theory of positive emotions. American Psychologist, 56(3), 218-226. http://doi:10.1037/0003-066X.56.3.218

Fredrickson, B. L. (2004). The broaden-and-build Theory of positive emotions. Philosophical Transactions of The Royal Society B, 359, 1367-1377. https:// doi.org/10.1098/rstb.2004.1512

Georgianni, S. K., \& Babatisikou, F. P. (2014). Psychological aspects in chronic renal failure. Health Science Journal, 8(2), 205-214.

Higa, K., Kost, M. T., Soares, D. M., Morais, M. C., \& Polins, B. R. G. (2008). Qualidade de vida de pacientes portadores de insuficiência renal crônica em tratamento de hemodiálise. Acta Paulista de Enfermagem, 21(n. esp.), 203-206. https://doi:10.1590/S0103-21002008000500012

Hutz, C. S., Nunes, C. H., Silveira, A. D., Serra, J., Anton, M., \&Wieczorek, L. S. (1998). O desenvolvimento de marcadores para a avaliação da personalidade no modelo dos cinco grandes fatores. Psicologia: Reflexão e Crítica, 11(2), 395-411.

Kavanagh, D. J., Gooley, S., \& Wilson, P. H. (1993). Prediction of adherence and control in diabetes. Journal of Behavioral Medicine, 16, 509-522. https://doi:10.1007/BF00844820

Kusumoto, L., Marques, S., Haas, V. J., \& Rodrigues, R. A. P. (2007). Adultos e idosos em hemodiálise: Avaliação da qualidade de vida relacionadaà saúde. Acta Paulista de Enfermagem, 21, 152-159. https://doi:10.1590/S0103-21002008000500003

Lucas, R. E., \& Diener, E. (2008). Personality and subjective well-being. In O. P. John, R. W. Robins, \& L. A. Pervin (Eds.), Handbook of personality: Theory and research (pp. 795-8140), New York: The Guilford Press.

Lyubomyrsky, S., King, L., \& Diener, E. (2005). The benefitis of frequente positive affect: Does happiness lead to success? Psychological Bulletin, 131(6), 803-855. https://doi:10.1037/0033-2909.131.6.803

Machado, G. S. (2006). Psicologia no transplante. In V. D. Garcia, M. A. Filho, J. Neumann, \& J. O. M. Pestana (Eds.), Transplante de órgãos e tecidos (pp. 76-81). São Paulo, SP: Segmento Farma.

Marshall, G. N., Wortman, C. B., Kusulas, J. W., Hervig, L. K., \& Vickers Jr., R. R. (1992). Distinguishing optimism from pessimism: Relations to fundamental dimensions of mood and personality. Journal of Personality and Social Psychology, 62, 1067-1074. https://doi:10.1037/0022-3514.62.6.1067

Martins, M. R. I., \& Cesarino, C. B. (2005). Qualidade devida de pessoas com doença renal crônica em tratamento hemodialítico. Revista Latino-americana de Enfermagem, 13(5), 670-676. https://doi:10.1590/S0104-11692005000500010

McCrae, R. R., \& Costa, P. T., Jr. (1997). Personality trait structure. American Psychologist, 52(5), 509-516. https://doi:10.1037/0003-066X.52.5.509

Nascimento, C. D., \& Marques, I. R. (2005). Intervenções de Enfermagem nas complicações mais frequentes durante a sessão de hemodiálise: Revisão da literatura. Revista Brasileira de Enfermagem, 58(6), 719-722. http://doi:10.1590/S0034-71672005000600017

Nunes, C. H. S., Hutz, C. S., \& Giacomoni, C. H. (2009). Associação entre bem-estar subjetivo e personalidade no modelo dos cinco grande fatores. Avaliação Psicológica, 8(1), 99-108.

Pereira, L. C., Chang, J., Fadil-Romao, M. A., Abensur, H., Araujo, M. R. T., Noronha, I. L., Campagnari, J. C., \& Romao, J. E., Jr. (2003). Qualidade de vida relacionada à saúde em paciente transplantado renal. Jornal Brasileiro de Nefrologia, 25(1), 10-16.

Pressman, S. D., \& Cohen, S. (2005). Does positive affect influence health? Psychological Bulletin, 131(6), 925-971. http://doi:10.1037/0033-2909.131.6.925

Ravagnani, L. M. B., Domingos, N. A. M., \& Miyazaki, M. C. O. S. (2007). Qualidade de vida e estratégias de enfrentamento em pacientes submetidos a transplante renal. Estudos de Psicologia, 12(2), 177-184. https://doi:10.1590/S1413-294X2007000200010

Rebollo, P., Ortega, F., Baltar, J. M., Badía, X., Alvarez-Ude, F., Díaz-Corte, C, Naves, M., Navascúes, R. A., Ureña, A., \& Alvarez-Grande, J. (2000). Health related quality of life (HRQOL) of kidney transplanted patients: variables that influence it. Clinical Transplantation, 14, 199-207. https://doi:10.1034/j.1399-0012.2000.140304.x

Romão, J. E., Jr. (2004). Doença renal crônica: Definição, epidemiologia e classificação. Jornal Brasileiro de Nefrologia, 26(3), 1-3.

Scheier, M. F, \& Carver, C. S. (1985). Optimism, coping, and health: Assessment and implications of generalized outcome expectancies. Health Psychology, 4(3), 219-247. https://doi:10.1037/0278-6133.4.3.219

Scheier, M. F., \& Carver, C. S., (1987). Dispositional optimism and physical well-being: The influence of generalized outcome expectancies on health. Journal of Personality, 55(2), 169-210. https://doi:10.1111/j.1467-6494.1987.tb00434.x 
Scheier, M. F., Magovern, G. J., Abott, R.A., Owens, K.A. M., J. F. Owens, Lefebvre, R. C., \& Carver, C. S. (1989). Dispositional Optimism and recovery from coronary artery bypass surgery: The beneficial effects on physical and psychological well-being. Journal of Personality and Social Psychology, 57(6), 1024-1040. https://doi:10.1037/0022-3514.57.6.1024

Scheier, M. F., Weintraub, J. K., \& Carver, C. S. (1986). Coping with stress: Divergent strategies of optimists and pessimists. Journal of Personality and Social Psychology, 51(6), 1257-1264. https:// doi:10.1037/0022-3514.51.6.1257

Seligman, M. E. P., Steen, T. A., Park, N., \& Peterson, C. (2005). Positive psychology progress: empirical validation of interventions. American Psychologist, 60(5), 410-421. https://doi:10.1037/0003-066X.60.5.410

Sesso, R. C., Lopes, A. A., Thomé, F. S., Lugon, J. R., \& Martins, C. T. (2017). Brazilian chronic dialysis Survey 2016. Brazilian Journal of Nephrology, 39(3), 261-266.

Sharpe, J. P. Martin, N.R., \& Roth, K. A. (2011). Optimism and the big five factors of personality: beyond neuroticism and extraversion. Personality and Individual Differences, 51, 946-951. https://doi:10.1016/j.paid.2011.07.033

Snyder, C. R., \& Lopez, S. J. (2009). Psicologia positiva: Uma abordagem científica e prática das qualidades humanas. Porto Alegre, RS: Artmed.

Stanton, A. L., Revenson, T. A., \& Tennen, H. (2007). Health psychology: Psychological adjustment to chronic disease. Annual Review of Psychology, 58(13), 565-592. https://doi:10.1146/annurev.psych.58.110405.085615

Stasiak, C. E. S., Bazan, K. S., Schuinski, A. F. M., \& Baroni, G. (2014). Prevalência de ansiedade e depressão e suas comorbidades em pacientes com doença renal crônica em hemodiálise e diálise peritoneal. Jornal Brasileiro de Nefrologia, 36(3), 325-331.

Steel, P., Schmidt, J., \& Shultz, J. (2008). Refining the relationship between personality and subjective well-being. Psychological Bulletin, 134(1), 138-161. https://doi:10.1037/0033-2909.134.1.138

Thomas, C. V., \&Alchieri, J. C. (2005). Qualidade de vida, depressão e características da personalidade em pacientes submetidos à hemodiálise. Avaliação Psicológica, 4(1), 57-64.

Vilhena, E., Pais-Ribeiro, J., Silva, I., Pedro, L., Meneses, R. F., Cardoso, H., Silva, A. M., \& Mendonça, D. (2014). Optimism and quality of life in Portuguese chronic patients: moderator/mediator? Revista da Associação Médica Brasileira, 60(4), 373-380. https:// doi:10.1590/1806-9282.60.04.017

Williams, D. G. (1992). Dispositional optimism, neuroticism, and extraversion. Personality and Individual Differences, 13, 475-477. https://doi:10.1016/0191-8869(92)90076-2

Wrosch, C., \& Scheier, M. F. (2003). Personality and quality of life: The importance of optimism and goal adjustment. Quality of Life Research, 12 (suppl 1), 69-72. https:// doi:10.1023/A:1023529606137

Zanon, C., Bastianello, M. R., Pacico, J. C., \& Hutz, C. S. (2013a). Desenvolvimento e validação de uma escala de afetos positivos e negativos. Psico-USF, 18(2), 193-202. https:// doi:10.1590/S1413-82712013000200003

Zanon, C., Bastianello, M. R., Pacico, J. C., \& Hutz, C. S. (2013b). Relationships between positive and negative affect and thefive factors of personalityina Brazilian sample.Paidéia,23(56),285-292.http://doi:10.1590/1982-43272356201302

\section{Renata Cipriano de Oliveira}

Mestre em Psicologia Aplicada pela Universidade Federal de Uberlândia, Uberlândia - MG. Brasil.

E-mail: renatinha.cipriano@gmail.com

(D) https://orcid.org/0000-0002-7625-9399

\section{Joaquim Carlos Rossini}

Docente do Programa de Pós-Graduação em Psicologia da Universidade Federal de Uberlândia, Uberlândia-MG. Brasil.

E-mail: jrossini@ufu.br

(D) https://orcid.org/0000-0001-6703-7770

\section{Renata Ferrarez Fernandes Lopes}

Professora titular do Instituto de Psicologia e do Programa de Pós-Graduação em Psicologia da Universidade Federal de Uberlândia, Uberlândia - MG. Brasil.

E-mail: rfernandeslopes@ufu.br

(D) https://orcid.org/0000-0002-5577-6450 
Psicologia: Ciência e Profissão 2020 v. 40, e209637, 1-16.

Endereço para envio de correspondência:

Av. Ana Godoy de Souza, 1983, apto. 104, Santa Mônica. CEP: 38408-290. Uberlândia - MG. Brasil.

Recebido 27/07/2018

Aceito 18/02/2019

Received $27 / 07 / 2018$

Approved 18/02/2019

Recibido $27 / 07 / 2018$

Aceptado 18/02/2019

Como citar: Oliveira, R. C., Rossini, J. C., \& Lopes, R. F. F. (2020). Otimismo Disposicional, Afetos e Personalidade em Pacientes com Doença Renal Crônica. Psicologia: Ciência e Profissão, 40, 1-16.

https://doi.org/10.1590/1982-3703003209637

How to cite: Oliveira, R. C., Rossini, J. C., \& Lopes, R. F. F. (2020). Dispositional Optimism, Affects and Personality in Patients with Chronic Renal Disease. Psicologia: Ciência e Profissão, 40, 1-16.

https://doi.org/10.1590/1982-3703003209637

Cómo citar: Oliveira, R. C., Rossini, J. C., \& Lopes, R. F. F. (2020). Optimismo Disposicional, Afectos y Personalidad en Pacientes com Enfermedad Renal Crónica. Psicologia: Ciência e Profissão, 40, 1-16.

https://doi.org/10.1590/1982-3703003209637 